\title{
Treatment of hepatocellular carcinoma in compensated cirrhosis with radio-frequency thermal ablation (RFTA): a prospective study
}

\author{
Calogero Cammà ${ }^{1,2, *}$, Vito Di Marco ${ }^{1}$, Ambrogio Orlando ${ }^{3}$, Luigi Sandonato ${ }^{4}$, Andrea Casaril ${ }^{5}$, \\ Piero Parisi ${ }^{1}$, Silvia Alizzi ${ }^{1}$, Elio Sciarrino ${ }^{3}$, Roberto Virdone ${ }^{3}$, Salvatore Pardo ${ }^{6}$, \\ Danilo Di Bona ${ }^{1,2}$, Anna Licata ${ }^{1}$, Federica Latteri ${ }^{1}$, Giuseppe Cabibbo ${ }^{1}$, Giuseppe Montalto ${ }^{7}$, \\ Mario Adelfio Latteri ${ }^{4}$, Nicola Nicoli ${ }^{8}$, Antonio Craxì ${ }^{1}$, Unità Interdipartimentale Neoplasie \\ Epatiche (U.I.N.E) Group \\ ${ }^{1}$ Cattedra e Unità Operativa di Gastroenterologia, University of Palermo, Palermo, Italy \\ ${ }^{2}$ IBIM, Consiglio Nazionale delle Ricerche, Palermo, Italy \\ ${ }^{3}$ Divisione di Medicina, Ospedale 'V. Cervello', Palermo, Italy \\ ${ }^{4}$ Dipartimento di Oncologia, Cattedra di Chirurgia Oncologica, University of Palermo, Palermo, Italy \\ ${ }^{5}$ Dipartimento di Chirurgia e Scienze Gastroenterologiche, University of Verona, Verona, Italy \\ ${ }^{6}$ Dipartimento di Radiologia 'P. Cignolini', University of Palermo, Palermo, Italy \\ ${ }^{7}$ Dipartimento di Medicina Interna, University of Palermo, Palermo, Italy \\ ${ }^{8}$ Dipartimento di Chirurgia, Ospedale 'V. Cervello', Palermo, Italy
}

Background/Aims: To assess the effectiveness and the safety of radio-frequency thermal ablation (RFTA) in patients with hepatocellular carcinoma (HCC) $\leq 5 \mathrm{~cm}$ in compensated cirrhosis.

Methods: A cohort of 202 consecutive patients (165 Child-Pugh class A and 37 class B) was prospectively assessed. A single lesion was observed in $160 / 202(79.2 \%)$, two lesions in 29/202 (14.3\%), and three lesions in 13/202 $(6.4 \%)$ of patients.

Results: Sixty-seven patients died. Survival rates were $80 \%$ at 12 months, $67 \%$ at 24 months and $57 \%$ at 30 months (Child-Pugh A 59\% and Child-Pugh B 48\%). By Cox regression analysis, survival was independently predicted by serum albumin levels $\geq 35 \mathrm{~g} / \mathrm{L}$, platelet count $\geq 100.000 / \mathrm{mmc}$, tumor size $\leq 3 \mathrm{~cm}$, complete response at 1 month and Barcelona Clinic Liver Cancer (BCLC) staging classification. Overall recurrence rates were 22, 38, and 44\% at 12, 24, and 30 months, respectively. One procedure-related death occurred. The proportion of major complications after treatment was $3.9 \%$.

Conclusions: A complete response after RFTA significantly increases survival. The longest survival is obtained in the presence of $\mathrm{HCC} \leq \mathbf{3} \mathrm{cm}$ and of higher baseline albumin levels and platelet counts. BCLC staging classification is able to discriminate patients with good or poor prognosis.

(C) 2005 European Association for the Study of the Liver. Published by Elsevier B.V. All rights reserved.

Received 12 August 2004; received in revised form 24 November 2004; accepted 29 November 2004; available online 30 January 2005

* Corresponding author. Address: Via A. De Gasperi 50, 90100 Palermo, Italy. Tel.: +39091655 3248; fax: +390916809122.

E-mail address: camma@ibim.cnr.it (C. Cammà).

Abbreviations: RFTA, Percutaneous radio-frequency thermal ablation; HCC, Hepatocellular carcinoma; CI, Confidence interval.

\section{Introduction}

The 5-year survival rate of patients with hepatocellular carcinoma $(\mathrm{HCC}) \leq 5 \mathrm{~cm}$ undergoing surgical or percutaneous ablation is lower than $50 \%$ because of the high risk of local recurrence particularly after percutaneous ablation, as well as the occurrence of new lesions in the liver even though treatment has been considered potentially curative 
[1-3]. In 2000, the European Association for the Study of the Liver (EASL) Monothematic Conference on Clinical Management of HCC [4] concluded that radio-frequency thermal ablation (RFTA) was not recommended as the standard percutaneous technique because of its higher rate of side effects and lower applicability of treatment due to tumor location, when compared to percutaneous ethanol injection (PEI). Since then, a small randomized controlled trial (RCT) showed that RFTA was superior to PEI with respect to local recurrence-free rates [5].

The primary aim of this prospective study was to assess the effectiveness and the safety of RFTA and to identify predictors of survival by multivariate analysis in a cohort of patients with $\mathrm{HCC} \leq 5 \mathrm{~cm}$ in compensated cirrhosis.

\section{Patients and methods}

\subsection{Patients}

From January 1999 to September 2003, 531 consecutive patients with HCC $\leq 5 \mathrm{~cm}$ were screened and 202 were treated by RFTA at the three referral speciality units involved in the study. We used the following inclusion criteria for RFTA: (a) age $>65 \mathrm{yr}$, or age $<65 \mathrm{yr}$ in patients who refused surgery or orthotopic liver trasplantation or with associated diseases contraindicating surgery; (b) a single HCC $5 \mathrm{~cm}$ in diameter or smaller or as many as three HCCs each $3 \mathrm{~cm}$ in diameter or smaller; (c) cirrhosis classified as Child-Pugh class A or B [6]; (d) absence of portal vein thrombosis or extrahepatic metastases; (e) platelet count $>40,000 / \mathrm{mm}^{3}$; prothrombin time ratio $>40 \%$. Exclusion criteria were age over 75 years, tumors $>5 \mathrm{~cm}$, extrahepatic metastases, portal vein thrombosis, ascites, or dilated bile ducts, cardiac arrhythmia. No exclusion criteria were related to the location of tumors for all patients in Child-Pugh class A. By contrast, patients in Child-Pugh B class in whom the tumor was not treatable by percutaneous approach were excluded from RFTA.

We used a commercial ultrasound (US) scanner (ESAOTE biomedica AU5) with a guide device. Before starting the procedure, all the patients were staged with spiral computed tomography (CT) scan, alfa-fetoprotein (AFP), and chest X-ray. The mean time elapsed between HCC diagnosis and the RFTA procedures was 1 month.

The study was approved by the local ethics committee and a written informed consent was obtained from all patients.

\subsection{Outcomes and follow-up}

One month after RFTA, a spiral CT scan was repeated and the AFP level was measured. Complete response was defined as no evidence of neoplastic disease and no evidence of new lesions at spiral CT one month after treatment. When there was evidence of tumor persistence, a second RFTA was performed. Treatment failure was defined as the presence of viable tumor at the end of therapy. The follow-up protocol included clinical assessment, measurements of liver function by Child-Pugh score, routine laboratory investigations, AFP level, US examination every 3 months, and abdominal spiral CT scan every 6 months.

Local recurrence, after total necrosis by spiral CT scan, was defined as the development of tumor staining at the margin of the tumor or enlargement of the tumor on a follow-up spiral CT. When an enlargement of the size of lesion, or a new lesion, was observed during US examination or an increase of $100 \mathrm{ng} / \mathrm{mL}$ AFP levels with respect to previous assay was found, a CT scan was performed. If local recurrence was detected, RFTA was repeated.

A separate new lesion in the liver (i.e. distant recurrence) was considered to have developed when there was the appearance of another focal lesion distinct from the treated one (i.e. a new nodule that arose more than $2 \mathrm{~cm}$ from the original lesion). In this case, a second RFTA was undertaken. Patients who developed diffuse HCC (i.e. more than 3 hepatic lesions) were not retreated.

\subsection{RFTA technique}

All treatments were performed only on inpatients under conscious sedation. RFTA was performed under ultrasound guidance with a $50 \mathrm{~W}$ electrical generator (Model 500 L; RITA Medical System, Mountain View, Calif.) connected to an expandable 15-gauge, $25.0-\mathrm{cm}$-long electrode with a 1.0-cm-long exposed tip (expandable by means of four hooks) until December 31 1999, and then with a $150 \mathrm{~W}$ generator (Model $1500 \mathrm{~L}$; RITA Medical System, Mountain View, Calif.) connected to an expandable 1514-gauge electrode with a 2.0-cm-long exposed tip (expandable by means of seven, or nine, hooks).

This electrode was placed into the center of the lesion maintaining the temperature of the needle tip at $100^{\circ} \mathrm{C}$ or more for $10-12 \mathrm{~min}$. When necessary due to tumor size, several insertions of the needle electrodes to treat overlapping zones were needed to complete the procedure, though never more than three insertions per procedure. Inclusion criteria for RFTA during laparotomy were: (a) cirrhosis Child-Pugh class A and (b) any location of tumors that precluded the percutaneous approach.

\subsection{Statistical evaluation}

The primary outcome of the study was overall survival. The secondary outcomes were: (a) overall recurrence defined as any appearance of the tumor (i.e. local recurrence or separate new lesions in the liver or extrahepatic metastases) during follow-up; (b) development of separate new lesions in the liver; (c) local recurrence. The Kaplan-Meier method [7] was used to estimate survival, overall recurrence, development of separate new lesions and of local recurrence. Differences in the survival rate were assessed by the log-rank test. The observed survival time was the interval between starting RFTA and death or the final follow-up. For overall recurrence, the tumor-free time was the interval between no evidence of neoplastic disease at spiral CT one month after the treatment and the appearance of the tumor. In this analysis, the follow-up of patients dying without recurrence was censored at the time of death, and these patients were classified as cancer-free. The following variables at baseline were considered for univariable analysis: participating centres, age, sex, anti$\mathrm{HCV}$ positivity, $\mathrm{HBsAg}$ positivity, alcohol abuse, bilirubin level, albumin level, prothrombin activity, platelet count, creatinine levels, tumor size, number of neoplastic lesions, AFP level, complete response to treatment, and Barcelona Clinic Liver Cancer (BCLC) staging classification. Variables with a $P$ value $<0.05$ at univariable analysis were included in the final multivariable model. The Cox model [8] was used to identify the risk factors for overall survival, appearance of separate new lesions and occurrence of local recurrence in a multiple regression analysis. All analyses were conducted with SAS [9].

\section{Results}

\subsection{Features of patients at baseline}

Seventy-four subjects out of 202 (36.6\%) were over 70 years of age. In all patients, HCC was associated with cirrhosis. Diagnosis of HCC was biopsy-proven in 158/202 subjects $(78.2 \%)$ and based on noninvasive diagnosis of HCC in 44/202 (21.8\%), according to international criteria [4]. The median value of AFP was $18 \mathrm{ng} / \mathrm{mL}$. Sixteen subjects only had AFP value $>400 \mathrm{ng} / \mathrm{mL}$.

A single lesion was observed in $79.2 \%$, two lesions in $14.3 \%$ and three lesions in $6.4 \%$ of patients (Table 1). The tumor size was $\leq 3 \mathrm{~cm}$ in $165 / 202(81.6 \%)$ of the patients. Only in $5 / 202(2.4 \%)$ a negligible amount of ascites was detected by ultrasound. At presentation 85 out of 202 
Table 1

Baseline features of 202 patients with hepatocellular carcinoma in compensated cirrhosis treated with RFTA according to Child-Pugh class

\begin{tabular}{|c|c|c|c|}
\hline Characteristics & $\begin{array}{l}\text { Child-Pugh A } \\
(n=165)\end{array}$ & $\begin{array}{l}\text { Child-Pugh B } \\
(n=37)\end{array}$ & $P$ Value \\
\hline Age (yrs) & $66.8 \pm 8.2$ & $67.4 \pm 6.9$ & 0.61 \\
\hline $\begin{array}{l}\text { Male sex-no. } \\
(\%)\end{array}$ & $108(65.4)$ & $23(62.1)$ & 0.70 \\
\hline $\begin{array}{l}\text { Anti-HCV posi- } \\
\text { tivity-no. }(\%)\end{array}$ & $119(72.1)$ & $23(62.1)$ & 0.23 \\
\hline $\begin{array}{l}\text { HbsAg positiv- } \\
\text { ity-no. }(\%)\end{array}$ & $26(15.7)$ & $11(29.7)$ & 0.04 \\
\hline Alcohol abuse & $16(9.6)$ & $5(13.5)$ & 0.99 \\
\hline $\begin{array}{l}\text { Alfa-Fetoprotein } \\
(\mathrm{ng} / \mathrm{mL})\end{array}$ & $128 \pm 364.5$ & $419.9 \pm 922.9$ & 0.43 \\
\hline $\operatorname{PLT} \times 10^{3} / \mathrm{mmc}$ & $113.8 \pm 34.4$ & $96.5 \pm 41.5$ & 0.02 \\
\hline $\begin{array}{l}\text { Serum albumin } \\
(\mathrm{g} / \mathrm{L})\end{array}$ & $37.9 \pm 4.7$ & $33.8 \pm 5.4$ & 0.0001 \\
\hline $\begin{array}{l}\text { Prothrombin } \\
\text { activity }(\%)\end{array}$ & $86.8 \pm 16.8$ & $74.6 \pm 16.4$ & 0.008 \\
\hline $\begin{array}{l}\text { Serum bilirubin } \\
(\mathrm{mg} / \mathrm{dl})\end{array}$ & $1.10 \pm 0.6$ & $1.85 \pm 0.9$ & 0.0001 \\
\hline $\begin{array}{l}\text { Creatinine levels } \\
(\mathrm{mg} / \mathrm{dl})\end{array}$ & $0.94 \pm 0.37$ & $0.89 \pm 0.25$ & 0.56 \\
\hline $\begin{array}{l}\text { Esophageal vari- } \\
\operatorname{ces}^{\mathrm{a}}-\text { no. }(\%)\end{array}$ & $62 / 130(47.6)$ & $14 / 30(46.6)$ & 0.75 \\
\hline \multicolumn{4}{|c|}{ Number of lesion-no. (\%) } \\
\hline Single lesion & $126(76.3)$ & $34(91.8)$ & 0.08 \\
\hline Two lesions & $26(15.7)$ & $3(8.1)$ & \\
\hline Three lesions & $13(7.8)$ & $0-$ & \\
\hline \multicolumn{4}{|c|}{ Tumor size-no. $(\%)$} \\
\hline$\leq 3 \mathrm{~cm}$ & $137(83.0)$ & $28(75.6)$ & 0.29 \\
\hline$>3 \mathrm{~cm}$ & $28(16.9)$ & $9(24.3)$ & \\
\hline \multicolumn{4}{|c|}{ BCLC Staging Classification-no. $(\%)$} \\
\hline A1 & $73(44.2)$ & $12(32.4)$ & 0.84 \\
\hline A2 & $43(26.0)$ & $10(27.0)$ & \\
\hline A3 & $10(6.0)$ & $12(32.4)$ & \\
\hline A4 & $26(15.7)$ & $1(2.7)$ & \\
\hline B & $13(7.8)$ & $2(5.4)$ & \\
\hline
\end{tabular}

Abbreviation: plus-minus values are means \pm SD.

a Assessed in 160 patients.

patients (42\%) belonged to the BCLC early (A1) stage. Only 15 patients (7.4\%) scored BCLC B, so for statistical purposes they were pooled with BCLC A4 in the survival analysis.

\subsection{Follow-up}

The mean length of follow-up after RFTA was 19.2 14.4 months (median 15 months, range 1-60 months) for the entire group. Twelve out of 202 subjects (5.9\%) were lost during follow-up visit. Their main baseline features did not differ significantly from the whole series of patients. During follow-up a total of 67 deaths occurred (Table 2). Among the 67 deaths only 8 patients (11.9\%) died cancer-free (i.e. without local recurrence, new lesions or distant metastases), while among the remaining 59 patients 11 subjects had a cancer progression and 48 subjects had a cancer recurrence. The final causes of death were liver failure in 45 patients,
Table 2

Follow-up of 202 patients with hepatocellular carcinoma in compensated cirrhosis treated with RFTA according to Child-Pugh class

\begin{tabular}{llll}
\hline Characteristics & $\begin{array}{l}\text { Child-Pugh } \\
\mathrm{A}(n=165)\end{array}$ & $\begin{array}{l}\text { Child-Pugh } \\
\mathrm{B}(n=37)\end{array}$ & $\begin{array}{l}\text { Total } \\
(n=202)\end{array}$ \\
\hline Complete response & & & \\
After one cycle (\%) & $135(81.8)$ & $29(78.3)$ & $164(81.1)$ \\
After two cycles (\%) & $17(10.3)$ & $6(16.2)$ & $23(11.3)$ \\
Treatment failure (\%) & $13(7.8)$ & $2(5.4)$ & $15(7.4)$ \\
Mean follow-up (mo \pm SD) & $19.5 \pm 13.8$ & $17.8 \pm 17.0$ & $19.2 \pm 14.4$ \\
Cancer recurrence-no. (\%) & $56(33.9)$ & $8(21.6)$ & $64(31.6)$ \\
Local recurrence-no. (\%) & $17(10.3)$ & $2(5.4)$ & $19(9.4)$ \\
New lesions-no. (\%) & $33(20)$ & $3(8.1)$ & $36(17.8)$ \\
Local recurrence plus new & $3(1.8)$ & $1(2.7)$ & $4(1.9)$ \\
lesion-no. $\%)$ & & & \\
Metastases-no. (\%) & $3(1.8)$ & $2(5.4)$ & $5(2.4)$ \\
RFTA retreatment-no. (\%) & $40(24.2)$ & $6(16.2)$ & $46(22.7)$ \\
New lesion & $26(15.7)$ & $5(13.5)$ & $31(15.3)$ \\
Local recurrence & $14(8.4)$ & $1(2.7)$ & $15(7.4)$ \\
Major complications-no. (\%) & $3(1.8)$ & $5(13.5)$ & $8(3.9)$ \\
Death-no. (\%) & $52(31.5)$ & $15(40.5)$ & $67(33.1)$ \\
\hline
\end{tabular}

Abbreviation: plus-minus values are means \pm SD.

variceal bleeding in 9 , spontaneous bacterial peritonitis in 4 , hemoperitoneum in 4 , cachexia in 3 , and unknown causes in 2.

The overall survival rates (Fig. 1) were $80 \%$ (95\% CI 74 $86), 67 \%$ (95\% CI 60-76), and 57\% (95\%CI 48-66) at 12 , 24 , and 30 months, respectively, without a significant difference among the three participating centres $(P=0.24$ by log-rank test). Survival was longer among Child-Pugh A class patients (83, 69 and 59\% at 12, 24, and 30 months, respectively) than among Child-Pugh B class patients (66, 60 , and $48 \%$ at 12,24 , and 30 months, respectively) ( $P 0.19$ by log-rank test). Survival was significantly longer in the subgroup of 85 potentially resectable patients, all in BCLC A1 stage, in comparison with all others $(P=0.004$ by logrank test).

Multivariate model, which considered baseline albumin levels, platelet counts, AFP, tumor size, complete response and BCLC staging classification, indicated that all variables, but AFP, were independent and significant predictors of overall survival (Table 3 ).

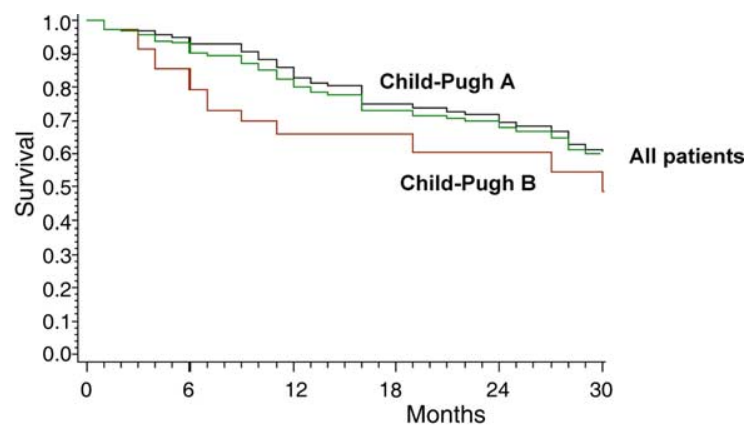

Fig. 1. Cumulative survival in all patients treated with radiofrequency thermal ablation for hepatocellular carcinoma and according to ChildPugh class A and Child-Pugh class B. [This figure appears in colour on the web.] 
Table 3

Adjusted relative risk for death in 202 patients with hepatocellular carcinoma in compensated cirrhosis treated with RFTA

\begin{tabular}{|c|c|c|c|c|c|}
\hline Variable & Code & $\beta$ & S.E. & $P$ Value & Relative risk ( $95 \% \mathrm{CI})$ \\
\hline Serum albumin & $\begin{array}{l}0:<35 \mathrm{~g} / \mathrm{L} \\
1: \geq 35 \mathrm{~g} / \mathrm{L}\end{array}$ & -0.56 & 0.26 & 0.03 & $0.57(0.33-0.96)$ \\
\hline Platelet count & $\begin{array}{l}0: \geqq 100.000 / \mathrm{mmc} \\
1:<100.000 / \mathrm{mmc}\end{array}$ & 0.57 & 0.27 & 0.03 & $1.76(1.03-3.03)$ \\
\hline Tumor diameter & $\begin{array}{l}0: \leq 3 \mathrm{~cm} \\
1:>3 \mathrm{~cm}\end{array}$ & 0.69 & 0.24 & 0.005 & $1.99(1.22-3.26)$ \\
\hline $\begin{array}{l}\text { BCLC staging classi- } \\
\text { fication }\end{array}$ & $\begin{array}{l}0: \mathrm{A} 1 \\
\text { 1: A2 } \\
\text { 2: A3 } \\
\text { 3: A4/B }\end{array}$ & 0.25 & 0.11 & 0.02 & $1.28(1.02-1.61)$ \\
\hline Complete response & $\begin{array}{l}0: \text { No } \\
\text { 1: Yes }\end{array}$ & -1.7 & 0.38 & 0.0001 & $0.18(0.085-0.39)$ \\
\hline
\end{tabular}

Adjusted for baseline alfa-fetoprotein levels.

In 64 patients, a cancer recurrence was observed during follow-up: 40 patients developed new lesions in the liver, 23 subjects developed a local recurrence, and in 5 subjects distant metastases were detected (Table 2). The cumulative overall recurrence-free rate (until separate new lesions, local recurrence or extrahepatic metastases occurred) is shown in Fig. 2. Overall recurrence rates were $22 \%$ at 12 months, $38 \%$ at 24 months and $44 \%$ at 30 months. Upon multivariate analysis, only platelet counts lower then $100.000 / \mathrm{mmc}$ (relative risk $2.90,95 \%$ CI $1.62-5.21$ ) was an independent risk factor for overall recurrence.

A total of 40 patients developed new lesions in the liver. The 12-, 24-, and 30-month rate of occurrence of separate new lesions was 13, 24, and 30\%, respectively (Fig. 3). In 31 out of 40 patients $(77.5 \%)$ who developed a separate new lesion, a second RFTA was performed. Only platelet counts lower then $100.000 / \mathrm{mmc}$ proved to be an independent risk factor for the occurrence of new lesions (relative risk 2.85, 95\% CI 1.46-5.53) by multivariate analysis.

In $92.5 \%$ (187/202 patients) of cases, a complete response was achieved; in $87.7 \%$ of these patients $(164 / 187)$ the complete tumor necrosis was achieved after one treatment; and in $12.3 \%$ (23/187), after two treatments.

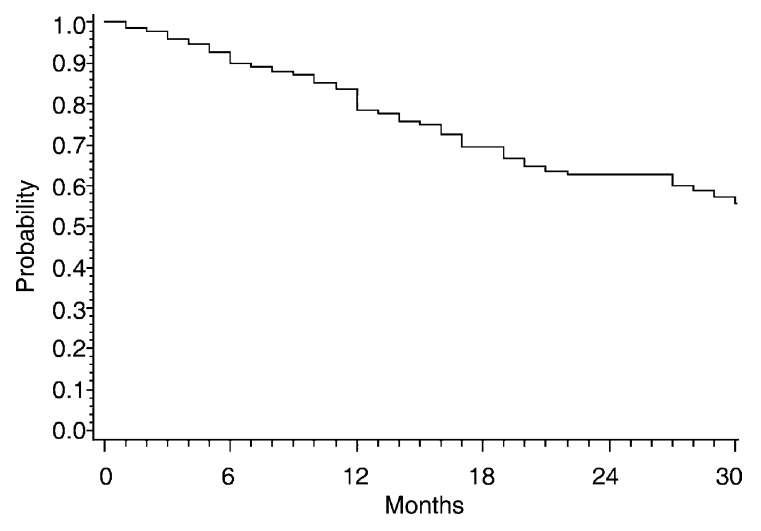

Fig. 2. Cumulative overall recurrence-free rate in all patients treated with radiofrequency thermal ablation for hepatocellular carcinoma.
In the remaining $7.5 \%(15 / 202)$ of patients, the tumor was still viable after two cycles of RFTA. We did not find a significant difference among the three participating centres in the rate of complete tumor necrosis $(P=0.42)$. In contrast, the overall rate of complete tumor necrosis achieved after one treatment was significantly higher for tumors $\leq 3 \mathrm{~cm}$ in diameter $(139 / 165,84.2 \%)$ than for those $>3 \mathrm{~cm}$ in diameter $(26 / 37,70.2 \%)(P=0.047)$.

In $23 / 187$ patients $(12.3 \%)$, a local recurrence was observed, and in 15 of those (65.2\%), a second RFTA was performed. The 12-, 24-, and 30-month rate of local recurrence was 10, 16, and 19\%, respectively (Fig. 3). None of the baseline variables analyzed were significantly associated with the occurrence of local recurrence.

\subsection{Treatment safety}

One treatment-related death was observed in a patient 4 weeks after RFTA due to liver failure following surgical resection for intestinal perforation. The proportion of major complications after treatment was $3.9 \%$. Three patients developed portal vein thrombosis soon after treatment. Other major reported complications were subcapsular hematoma (1 case), intrahepatic abscess ( 1 case) and pleural effusion ( 3 cases). The overall rate of post-treatment adverse

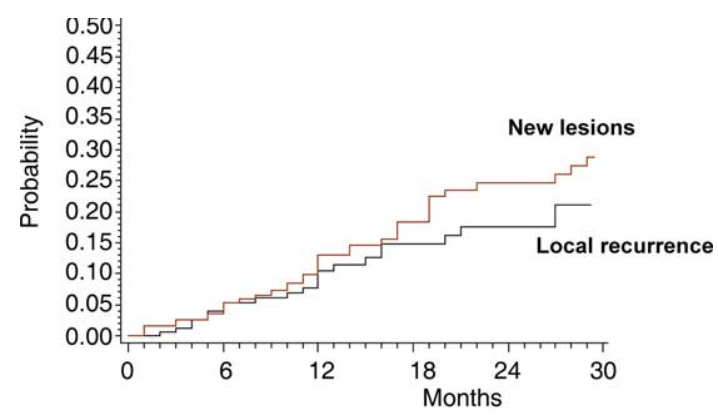

Fig. 3. Rate of occurrence of distant and local recurrence in patients with hepatocellular carcinoma treated with radiofrequency thermal ablation. [This figure appears in colour on the web.] 
events was significantly higher in Child-Pugh B class patients $(5 / 37,13.5 \%)$ than in those in Child-Pugh A class $(3 / 165,1.8 \%)(P<0.001)$.

None of the treated patients experienced either needletrack seeding or peritoneal hemorrhage. Minor complications were reported in 10/202 (4.9\%) patients. The three most frequent minor complications after treatment were pain requiring major analgesia, transient episode of fever and skin burns.

\subsection{Subgroup analysis of patients who underwent RFTA during laparotomy}

RFTA was performed during laparotomy in 52/202 patients $(25.7 \%)$, all in Child-Pugh A class. Their main baseline features did not differ significantly from the whole series of Child-Pugh A class patients. RFTA was performed during laparotomy because the tumor was not treatable by percutaneous approach (location near of main vascular vessels, portal branches or perihiliar hepatic region) in $10 / 52$ patients $(19.2 \%)$, because the tumor was located in the surface of the liver in 20/52 (38.4\%) and because tumor location was near the stomach, the colon, the gallbladder or the heart in $14 / 52$ subjects $(26.9 \%)$. In the remaining 8 patients $(15.3 \%)$, the nodule was preoperatively judged resectable, but RFTA was evaluated as safer than surgical resection during laparotomy. Treatment-related death as well as major complications were not observed. In all patients, treated during laparotomy a complete tumor necrosis was achieved. No significant difference was observed in the local recurrence rate between patients treated during laparotomy $(5 / 52 ; 9.6 \%)$ and those treated percutaneously $(18 / 150 ; 12 \%)(P=0.64)$. Overall survival did not differ significantly between Child-Pugh A class patients who underwent RFTA percutaneously and those who underwent RFTA during laparotomy $(P=0.34)$.

\section{Discussion}

To our knowledge, the findings in the current study, evaluating the impact of RFTA on a large cohort of patients with $\mathrm{HCC} \leq 5 \mathrm{~cm}$ in diameter in compensated cirrhosis, represents the largest prospective cohort study to date. In the present study, the 30-month overall survival rate was $57 \%$. This reported value for efficacy is in keeping with the 2-year survival of $63 \%$ observed in our previously published study, in which patients with similar characteristics were treated with PEI [10]. Only one small RCT reported that RFTA was superiot to PEI with respect to local recurrence-free survival rates [5].

Lencioni et al. [5] failed to identify any predictor significantly associated with survival after RFTA. We did not confirm the observation of Giovannini [11] suggesting a better survival after RFTA in patients who develop HCC after alcoholic cirrhosis compared to those in whom HCC occurs after viral cirrhosis. Our study shows that patients with HCC and no advanced liver disease, assessed by high baseline albumin and platelet levels, may benefit from RFTA treatment. The finding that high baseline albumin level was associated with improvement in survival is in agreement with the results of our previous study on PEI [10], as well as those of the study by Castellano [12]. Although a limitation to our study is the lack of data on direct assessment of portal hypertension [13], an interesting finding was the observation that platelet counts, a well known surrogate marker of clinically relevant portal hypertension, proved to be a strong predictor of survival. Finally, a cancer-related variable, such as the tumor size, emerges as an independent predictor for better survival upon multivariate analysis.

The antitumor effect of RFTA has been substantiated by this study showing a complete response rate of $92 \%$. This reported value for complete response is in keeping with the results reported in the literature, ranging from 85 [14] to $91 \%$ [5]. From the present study, it seems that overall survival strictly depends from the achievement of a complete tumor necrosis, suggesting that the achievement of a complete response should be carefully assessed by CT scan in all patients after RFTA. However, histologic data supporting radiologic assessment of complete response are lacking, suggesting that local recurrence could be interpreted as failure to provide adequate local control of HCC, particularly when viable tumor was detected within 6 months from treatment in spite of a radiologic assessment of complete response one month after treatment.

Our study shows that the BCLC staging classification sensitively identifies patients with HCC with a good or unfavorable prognosis. This clinical staging system is simple and represents a valuable tool for the prognostic evaluation of the patient, particularly in the setting of early tumors. It is important to underscore that the 12 Child-Pugh B7 patients classified as BCLC A1 included in this study were a very well compensated subgroup of subjects with normal serum bilirubin, serum albumin levels between 35 and $30 \mathrm{~g} / \mathrm{L}$ and prothrombin activity between 70 and $50 \%$, all without clinically relevant portal hypertension.

An important finding in the present study was the high rate of appearance of new lesions in the liver. The 3-year occurrence of new lesions in our series $(34 \%)$ was quite similar to that of our previous study on PEI (41\%), as well as that found in the study by Lencioni [5], in which the 2-year recurrence rate of patients treated by RFTA was $36 \%$. Only baseline platelet counts were found upon multivariate analysis to independently predict the development of new lesions in the liver.

In about $25 \%$ of patients, we were unable to perform RFTA by percutaneous approach due to tumor location. In these cases, RFTA was performed during laparotomy, thus increasing costs and limiting the applicability of RFTA, particularly when compared to PEI. However, treatmentrelated death as well as major complications were not 
observed in this subgroup of patients and the reported value for survival was similar to that of Child A class patients treated by a percutaneous approach, indicating that RFTA performed during laparotomy is a safe and efficacious procedure.

A recent large multicenter retrospective study [15] reported a low rate $(0.5 \%)$ of seeding after thermal ablation of HCC or colorectal metastasis. Indeed, Lencioni et al. [5] did not observe any case of tumor seeding after RFTA. Our study, confirming the observation of Lencioni and colleagues [5], provides further evidence that the risk of seeding is very low during follow-up.

In this study, one procedure-related death was observed and additional major complications occurred (portal vein thrombosis, pleural effusions, intrahepatic abscess and subcapsular hematoma) in about $4 \%$ of patients. We observed that the overall rate of post-treatment major adverse events was significantly higher in Child-Pugh B class patients than in those in Child-Pugh A class.

The available evidence is sufficient to conclude the following: (1) a complete response after RFTA significantly increases survival; (2) the overall survival rate after 30 monts of follow-up remains low (57\%) because of the high rate of recurrence; [3] a tumor size $>3 \mathrm{~cm}$ and low baseline albumin and platelet levels significantly increase the risk of death; BCLC staging classification is able to discriminate patients with good or poor prognosis; [4] the applicability of percutaneous RFTA may be restricted in about 25\% of patients by tumor location. RFTA performed during laparotomy is an effective and safe procedure.

\section{Acknowledgements}

The authors thank Warren Blumberg for his forbearance in editing the manuscript. Grant support: the full cost of the study was sustained by the institutions for which the authors work.

\section{Appendix A}

In addition to the authors, the following investigators, for the Unità Interdipartimentale Neoplasie Epatiche (U.I.N.E) Group, participated in this study:

Maurizio Soresi, Dipartimento di Medicina Interna, University of Palermo, Italy; Sergio Salerno, Dipartimento di Radiologia 'P. Cignolini', University of Palermo, Italy;
Daniela Cabibi, Dipartimento di Anatomia Patologica, University of Palermo, Italy; Ciro Marrone, Maddalena Albanese, Gaetano Pantalone Restivo, Divisione di Medicina, Ospedale 'V. Cervello', Palermo, Italy.

\section{References}

[1] Llovet JM, Burroughs A, Bruix J. Hepatocellular carcinoma. Lancet 2003;362:1907-1917.

[2] Llovet JM, Fuster J, Bruix J. Intention-to-treat analysis of surgical treatment for early hepatocellular carcinoma: resection versus transplantation. Hepatology 1999;30:1434-1440.

[3] Llovet JM, Beaugrand M. Hepatocellular carcinoma: present status and future prospects. J Hepatol 2003;38:S136-S149.

[4] Bruix J, Sherman M, Llovet JM, Beaugrand M, Lencioni R, Burroughs AK, et al. EASL panel of Experts on HCC. Clinical management of hepatocellular carcinoma. Conclusions of the Barcelona-2000 EASL conference. J Hepatol 2001;35:421-430.

[5] Lencioni RA, Allgaier HP, Cioni D, Olschewski M, Deibert P, Crocetti L, et al. Small hepatocellular carcinoma in cirrhosis: randomized comparison of radio-frequency thermal ablation versus percutaneous ethanol injection. Radiology 2003;228:235-240.

[6] Pugh RNH, Murray-Lyon IM, Dawson JL, Pietroni MC, Williams R. Transection of the esophagus for bleeding oesophageal varices. $\mathrm{Br}$ J Surg 1936;60:646-664.

[7] Kaplan EI, Meier P. Non-parametric estimation from incomplete observation. J Am Stat Assoc 1958;53:457-481.

[8] Cox DR. Regression models and life tables (with discussion). J R Stat Soc B 1972;34:187-220.

[9] SAS Technical Report, SAS/STAS software: changes and enhancement, release 6.07. Cary, NC: SAS Institute Inc; 1992.

[10] Orlando A, D'Antoni A, Camma C, Albanese M, Livraghi T, Torzilli G, et al. Treatment of small hepatocellular carcinoma with percutaneous ethanol injection: a validated prognostic model. Am J Gastroenterol 2000;95:2921-2927.

[11] Giovannini M, Moutardier V, Danisi C, Bories E, Pesenti C, Delpero JR. Treatment of hepatocellular carcinoma using percutaneous radiofrequency thermoablation: results and outcomes in 56 patients. J Gastrointest Surg 2003;7:791-796.

[12] Castellano L, Calandra M, Del Vecchio Blanco C, de Sio I. Predictive factors of survival and intrahepatic recurrence of hepatocellular carcinoma in cirrhosis after percutaneous ethanol injection-analysis of 71 patients. J Hepatol 1997;27:862-870.

[13] Llovet JM, Bru C, Bruix J. Prognosis of hepatocellular carcinoma: the BCLC staging classification. Semin Liver Dis 1999;19:329-338.

[14] Livraghi T, Goldberg SN, Lazzaroni S, Meloni F, Solbiati L, Gazelle GS. Small hepatocellular carcinoma: treatment with radiofrequency ablation versus ethanol injection. Radiology 1999;210: 655-661.

[15] Livraghi T, Solbiati L, Meloni MF, Gazelle GS, Halpern EF, Goldberg SN. Treatment of focal liver tumors with percutaneous radio-frequency ablation: complications encountered in a multicenter study. Radiology 2003;226:441-451. 